\title{
Isolated recurrence of acute myeloid leukemia in the cerebellum: illustrative case
}

\author{
Vikraant Kohli, BS, ${ }^{1}$ Michael T. Koltz, MD, ${ }^{1,2}$ and Ashwin A. Kamath, MD ${ }^{1,2}$ \\ ${ }^{1}$ Department of Neurosurgery, Dell Medical School, The University of Texas at Austin, Austin, Texas; and ${ }^{2}$ Department of Neurosurgery, Seton Brain and Spine Institute, \\ Austin, Texas
}

\begin{abstract}
BACKGROUND Myeloid sarcoma is a rare malignant hematopoietic neoplasm that arises at extramedullary sites. Although myeloid sarcoma may involve any organ, central nervous system (CNS) involvement is exceptionally rare. To date, few case reports and case series have described CNS myeloid sarcoma, the majority of which include peripheral disease.

OBSERVATIONS The authors present an illustrative case of an adult male with acute myeloid leukemia (AML) in remission relapsing with an isolated, diffuse myeloid sarcoma of the cerebellum. Magnetic resonance imaging showed posterior fossa crowding and diffuse enhancement within the cerebellar white matter without an apparent mass lesion. The patient required ventriculostomy due to obstructive hydrocephalus and ultimately suboccipital craniectomy with duraplasty due to posterior fossa compression. An open cerebellar biopsy revealed myeloid sarcoma. Peripheral studies did not meet the criteria for recurrent AML. The patient subsequently received high-dose systemic chemotherapy and has responded well to treatment.

LESSONS Myeloid sarcoma may be a neurosurgical lesion because it has the potential to cause mass effect with obstructive hydrocephalus requiring emergent cerebrospinal fluid diversion and possible decompression. The authors report a rare case of isolated recurrence of $A M L$ in the form of diffuse CNS myeloid sarcoma and describe the role of neurosurgery in its diagnosis and treatment.
\end{abstract}

https://thejns.org/doi/abs/10.3171/CASE21281

KEYWORDS myeloid sarcoma; granulocytic sarcoma; chloroma; acute myeloid leukemia; cerebellum; central nervous system

The spread of leukemia to the central nervous system (CNS) is a poor prognostic sign. ${ }^{1,2}$ In contrast to the pediatric population, the overall incidence of CNS involvement in adult patients with acute myeloid leukemia (AML) is uncertain. ${ }^{2,3}$ This lack of information has been attributed to lumbar puncture (LP) not being routinely performed in adults with $\mathrm{AML}$ when CNS signs and symptoms are not present. ${ }^{4}$ In a study of 3261 patients with AML, a total of 21 patients $(0.6 \%)$ had CNS involvement at the time of initial presentation, and 34 patients (2.9\%) had CNS involvement at relapse $(P<0.010)^{5}$

Myeloid sarcoma, also termed "granulocytic sarcoma" or "chloroma," is defined as a malignant hematopoietic neoplasm that arises at extramedullary sites. ${ }^{6}$ Myeloid sarcoma typically manifests as a solid tumor and is associated with a concurrent or previous diagnosis of AML. ${ }^{7}$ Although myeloid sarcoma may involve any organ, CNS involvement is exceptionally rare. A population-based study by Østgaard et al. ${ }^{8}$ found CNS myeloid sarcoma in only $0.4 \%$ of 2261 patients with AML. To date, only a handful of case reports and small case series have described CNS myeloid sarcoma. ${ }^{1,2,5,6,9-13}$ We present a unique case of an adult male with $A M L$ in remission relapsing with an isolated, diffuse myeloid sarcoma of the cerebellum.

\section{Illustrative Case}

A 49-year-old man with a past medical history of AML in remission presented to a regional hospital emergency department (ED) with 1 week of throbbing headaches, nausea, and "not being himself." His headaches were primarily frontal, constant, and gradually worsening. Moreover, his friends had noticed him speaking somewhat incoherently over the past week. He denied fevers, chills, vision changes, weakness, or loss of consciousness.

The patient had been diagnosed with AML 7 years prior and had received 2 rounds of induction chemotherapy (idarubicin + cytarabine) with intrathecal prophylaxis due to headache and an

ABBREVIATIONS AML = acute myeloid leukemia; CNS = central nervous system; CSF = cerebrospinal fluid; $C T=$ computed tomography; ED = emergency department; EVD = external ventricular drain; ICU = intensive care unit; $L P=$ lumbar puncture; $M P O=$ myeloperoxidase; MRI = magnetic resonance imaging INCLUDE WHEN CITING Published August 23, 2021; DOI: 10.3171/CASE21281

SUBMITTED May 7, 2021. ACCEPTED June 17, 2021.

(C) 2021 The authors, CC BY-NC-ND 4.0 (http://creativecommons.org/licenses/by-nc-nd/4.0/). 
inconclusive LP. At that time, the finding of his neuroimaging was normal. He also underwent bone marrow transplant complicated by graftversus-host disease of the eyes and liver. The patient had remained in remission until 18 months ago, when bone marrow studies demonstrated relapse. He was treated with cladribine, cytarabine, and filgrastim consolidation chemotherapy. His relapse was complicated by invasive fungal sinusitis with orbital involvement, requiring multiple sinus surgeries as well as repair for an iatrogenic cerebrospinal fluid (CSF) leak. Since the relapse, the patient was started on gilteritinib, a medication he continues to take at home. The patient also underwent bilateral cataract surgery, and his left eye is blind at baseline. He has no family history of brain or spine conditions.

On examination, the patient was alert, oriented, and able to follow commands. He was in visible discomfort from a headache. His speech was clear and fluent with the use of short sentences. His left pupil was opacified, his right pupil was $2-3 \mathrm{~mm}$, and his extraocular movements were intact. His face was symmetric, his tongue was midline, and he was able to move all extremities well. Furthermore, he exhibited $5 / 5$ strength and had no evidence of pronator drift. In the ED, noncontrast computed tomography (CT) of the head showed ventriculomegaly with relative sparing of the 4th ventricle, concerning for obstructive hydrocephalus (Fig. 1). The patient was subsequently transferred to our facility for neurosurgical evaluation. Upon arrival, a right frontal external ventricular drain (EVD) was placed at the bedside, and the patient was admitted to the intensive care unit (ICU).

Subsequent magnetic resonance imaging (MRI) with and without contrast revealed posterior fossa crowding, aqueductal obstruction, and cerebellar hypertrophy with diffuse nonspecific enhancement within the cerebellar white matter (Fig. 2). The results of complete blood count and CSF studies, including glucose, protein, cell counts, cultures, and cytology, were unremarkable. The differential diagnosis at this time included CNS recurrence of AML, autoimmune paraneoplastic cerebellitis, and cerebellar gangliocytoma. Over the next few days, the patient experienced a worsening headache and brief episodes of apnea, concerning for posterior fossa compression. As a result, on hospital day 4, he was taken to the operating room for suboccipital decompressive craniotomy, C1 laminectomy, and duraplasty. After CSF studies were resent, cytology results revealed rare immature myelomonocytic cells, suggestive of AML.

Subsequently, the patient required placement of a ventriculoperitoneal shunt after a series of unsuccessful EVD weans. The patient underwent a bone marrow biopsy, which revealed no evidence of
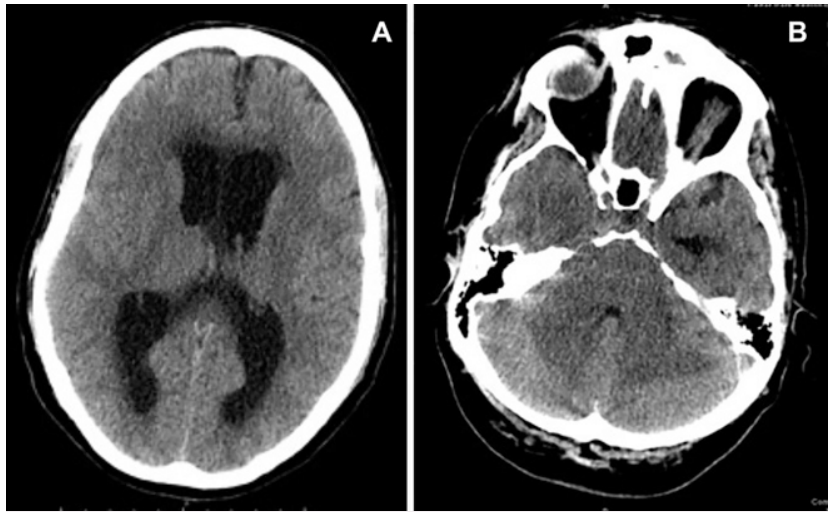

FIG. 1. A and B: Initial CT of the head shows ventriculomegaly with relative sparing of the 4 th ventricle, concerning for obstructive hydrocephalus.
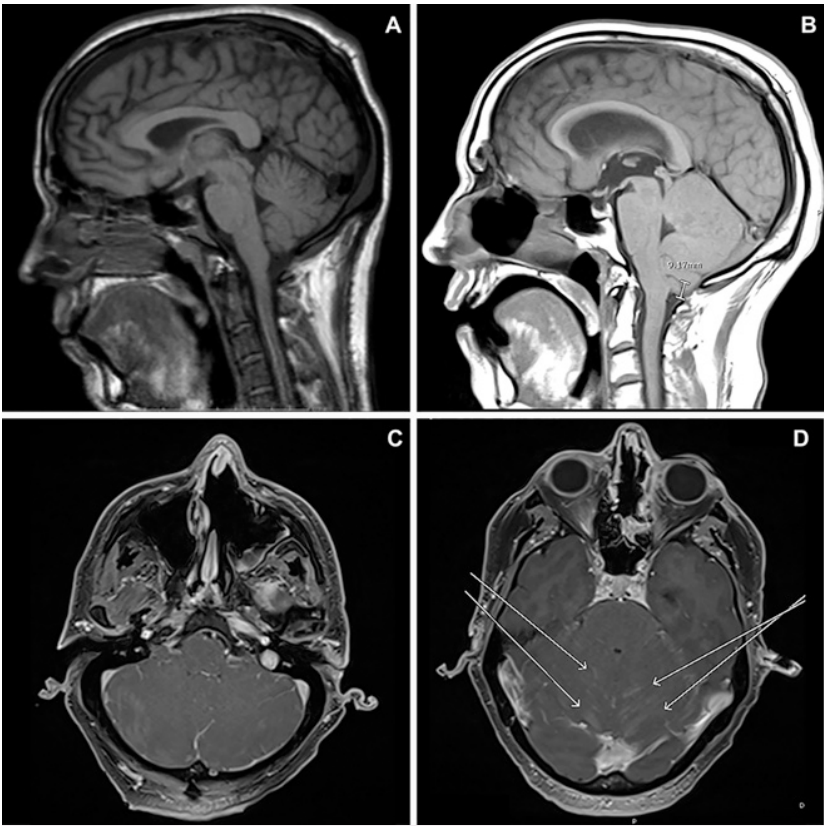

FIG. 2. Comparison of T1-weighted MRI of the brain from 7 years prior (A) and present T1-weighted MRI of the brain with gadolinium (B-D). Current MRI shows increased posterior fossa crowding, aqueductal obstruction, and cerebellar hypertrophy with diffuse nonspecific enhancement within the cerebellar white matter (arrows). Mild tonsillar herniation is also noted.

systemic AML recurrence. This result suggested possible isolated CNS recurrence of AML. Because other studies were mostly equivocal and this unusual presentation of recurrent AML would require intensive chemotherapy, the oncology team requested a cerebellar biopsy for definitive diagnosis. An open cerebellar biopsy was performed. The final pathology revealed a diffuse infiltrate of monocytic blasts that stained positive for CD43 and myeloperoxidase (MPO), consistent with myeloid sarcoma (Fig. 3).

The patient was initiated on high-dose systemic cytarabine chemotherapy for 4 total doses. On the day after chemotherapy completion, the patient experienced multiple episodes of seizure-like activity and nystagmus. Prompt MRI of the brain showed progressive edema in the brainstem, medial thalamic, and subcortical regions, concerning for disease progression versus treatment effect (Fig. 4A and B). In the following days, the patient's electroencephalogram revealed multiple focal seizures. Ultimately, the family elected to transition the patient from intubation to tracheostomy and continued observation at a long-term acute care facility.

At his follow-up visit, the patient demonstrated remarkable improvement. His most recent MRI scan 3 months after discharge showed a significant reduction in posterior fossa edema (Fig. 4C), an achievement of the lifesaving surgical goals. His tracheostomy and percutaneous endoscopic gastrostomy tube were subsequently removed. He is currently functioning well and requires minimal assistance with activities of daily living.

\section{Discussion}

\section{Observations}

In a review of 127 histologically confirmed CNS myeloid sarcoma cases, $61 \%$ of cases were secondary to or coincidental with 


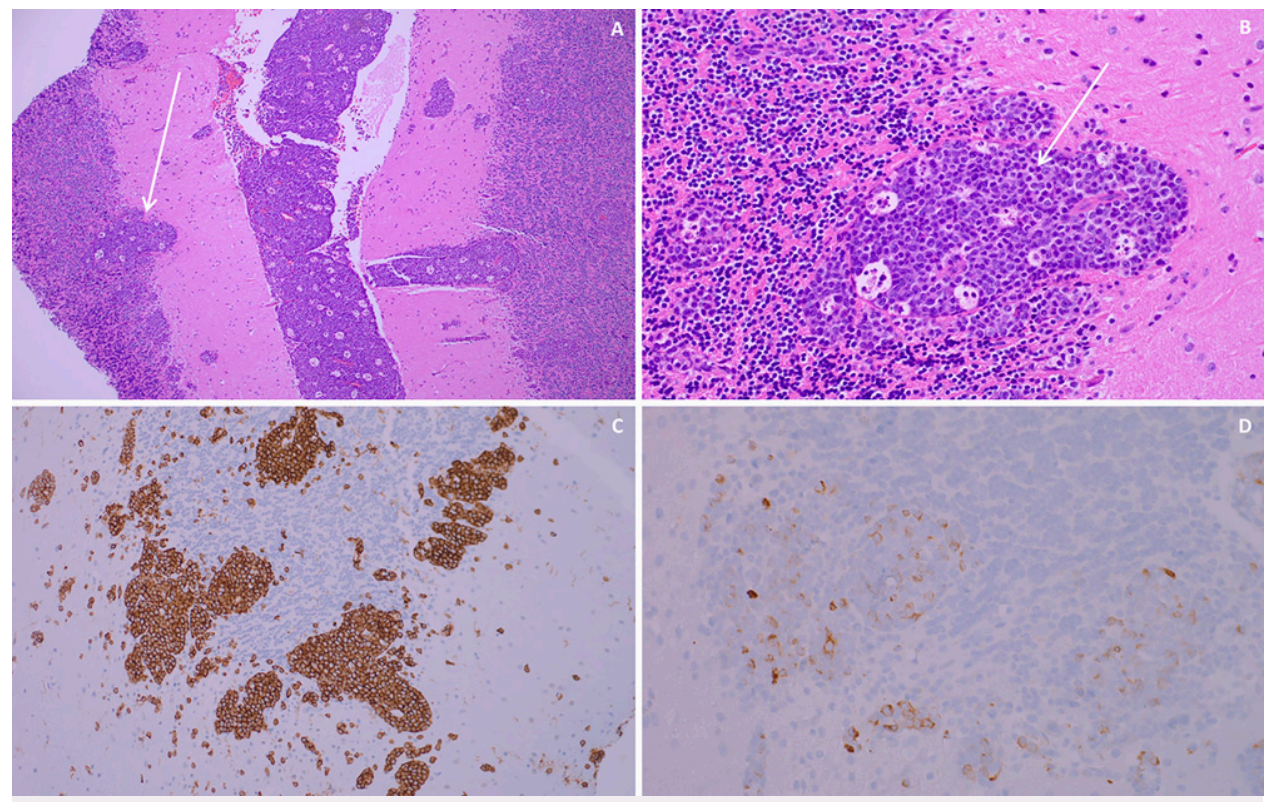

FIG. 3. Photomicrographs of biopsied cerebellum. A: Image at $100 \times$ magnification stained for hematoxylin and eosin (H\&E) shows clusters of atypical cells with monocytic differentiation (arrow) characteristic of myeloid sarcoma. B: Image at 400× magnification stained for H\&E shows atypical myeloid cells that contain heterogeneous amounts of cytoplasm and irregular nuclear contours (arrow). C: Image at $200 \times$ magnification stained for CD43. D: Image at 400× magnification stained for MPO.

hematological neoplasia, the most common being AML. ${ }^{1}$ Among these patients, CNS myeloid sarcoma was more common in the spinal cord than in the brain (54\% versus $40 \% ; P=0.033)$, with tumors primarily located in the epidural layer causing cord compression. ${ }^{1}$ In the brain, myeloid sarcoma more commonly involved the parenchyma (43\%) and dura $(41 \%) .^{1}$ Involvement of the cerebellum, as in our patient, has been reported in a minority of patients (17\%). ${ }^{1}$

The pathogenesis of CNS myeloid sarcoma is uncertain. ${ }^{11-13}$ One theory cited by Azzarelli et al. ${ }^{14}$ holds that leukemic cells in the bone marrow migrate through the Haversian canals toward the periosteum and infiltrate the perivenous adventitial tissue connecting the dura mater and subarachnoid space. Others have proposed that specific glycoproteins involved in cell adhesion, such as CD56, allow leukemic cells to invade and survive in extramedullary tissues. ${ }^{15}$ Pui et al. ${ }^{16}$ considered other mechanisms, including leukemic invasion of the CSF via the choroid plexus as well as leukemic cell growth along nerve roots leading to subarachnoid space infiltration through neural foramina.

In general, the diagnosis of CNS leukemia is made by the presence of leukemic blasts in CSF analysis after LP. ${ }^{17}$ Specific to CNS myeloid sarcoma, radiographic findings are variable or nonspecific and can mimic other intracranial pathologies. ${ }^{9}$ As a result, biopsy should be performed by an experienced neurosurgeon to confirm the diagnosis. ${ }^{18}$ The histopathologic morphology exhibits myeloid cell infiltration, whereas immunohistochemical staining has been shown to have many positive biomarkers, especially CD34, CD43, CD68, and MPO., ${ }^{9} 18$
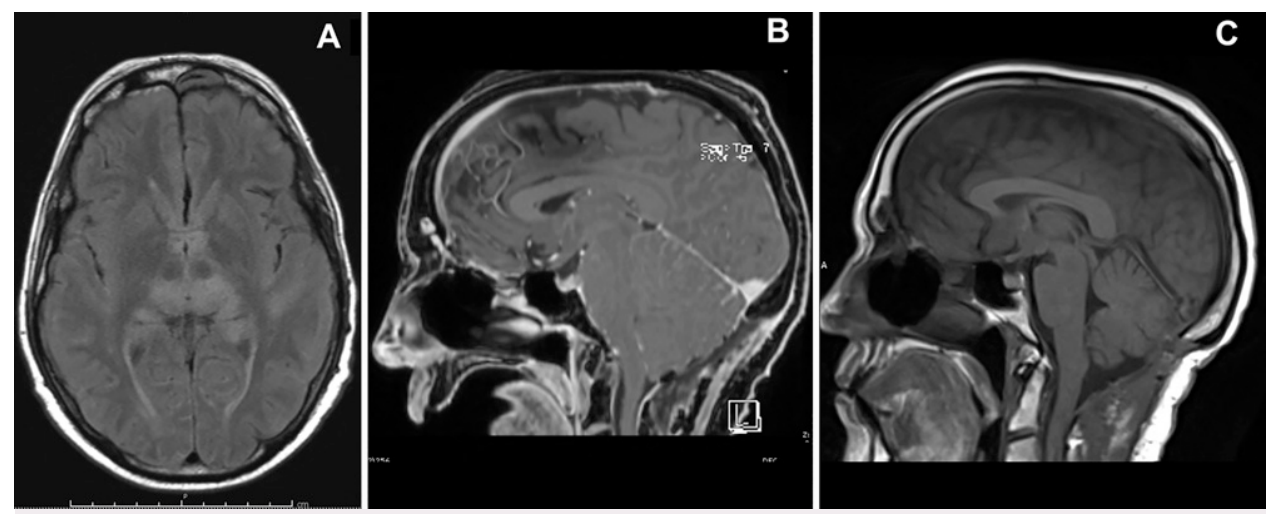

FIG. 4. A: Fluid-attenuated inversion recovery MRI of the brain shows progressive edema in the thalamic and subcortical regions. B: T1-weighted MRI of the brain with gadolinium shows severe cerebellar hypertrophy and brainstem compression. C: Follow-up visit T1-weighted MRI of the brain at 3 months shows significant improvement in posterior fossa edema. 
The standard therapy for myeloid sarcoma involves treatment of the underlying neoplasia. ${ }^{10}$ In adult patients with CNS involvement of AML, systemic therapy in addition to intrathecal chemotherapy and/or cranial irradiation is considered. ${ }^{5}$ No randomized controlled trials have been performed to compare the use of these treatment modalities in patients with CNS leukemia. Surgery has been reported to serve an important role in patients with myeloid sarcoma who are symptomatic due to mass effect of the lesion. ${ }^{7}$ Almond et al. ${ }^{7}$ recommended consideration of surgical excision or debulking in these patients before initiation of systemic therapy. A significant contrast to the literature in our case was the presence of considerable mass effect without an apparent mass lesion. Surgery for decompression, which was lifesaving for our patient, has been reported in a minority of patients with CNS myeloid sarcoma. ${ }^{1,9}$

In terms of prognosis, a review of 125 cases of CNS myeloid sarcoma reported complete resolution after therapy in $19.2 \%$ of patients. ${ }^{1}$ The overall survival after diagnosis of CNS myeloid sarcoma in 94 patients ranged from a few days to 114 months (mean, 14 months). ${ }^{1}$

\section{Lessons}

CNS involvement of myeloid sarcoma is exceptionally rare. This disease warrants consideration in patients with a history of hematopoietic neoplasm who present with CNS signs or symptoms despite the absence of peripheral disease. CNS myeloid sarcoma requires neurosurgical consideration because it has the potential to cause mass effect with obstructive hydrocephalus requiring emergent CSF diversion and possible decompression. In such cases, performing LP for diagnosis would be contraindicated. The diagnosis is confirmed by biopsy of the lesion, which should be performed in a timely manner. The authors aim to increase the awareness of isolated recurrence of AML in the form of CNS myeloid sarcoma and the role of neurosurgery in its diagnosis and treatment.

\section{References}

1. Olar A, Lapadat R, Davidson CJ, et al. Central nervous system involvement by myeloid sarcoma: a report of 12 cases and review of the literature. Clin Neuropathol. 2016;35(5):314-325.

2. Del Principe MI, Buccisano F, Soddu S, et al. Involvement of central nervous system in adult patients with acute myeloid leukemia: incidence and impact on outcome. Semin Hematol. 2018;55(4): 209-214.

3. Johnston DL, Alonzo TA, Gerbing RB, et al. Central nervous system disease in pediatric acute myeloid leukemia: a report from the Children's Oncology Group. Pediatr Blood Cancer. 2017;64(12):e26612.

4. Döhner H, Estey E, Grimwade D, et al. Diagnosis and management of AML in adults: $2017 \mathrm{ELN}$ recommendations from an international expert panel. Blood. 2017;129(4):424-447.

5. Alakel N, Stölzel F, Mohr B, et al. Symptomatic central nervous system involvement in adult patients with acute myeloid leukemia. Cancer Manag Res. 2017;9:97-102.

6. Palejwala AH, O'Connor KP, Shi H, Villeneuve L, Scordino T, Glenn CA. Chronic myeloid leukemia manifested as myeloid sarcoma: review of literature and case report. J Clin Neurosci. 2019;64: 269-276.
7. Almond LM, Charalampakis M, Ford SJ, Gourevitch D, Desai A. Myeloid sarcoma: presentation, diagnosis, and treatment. Clin Lymphoma Myeloma Leuk. 2017;17(5):263-267.

8. Østgaard LSG, Sengeløv H, Holm MS, et al. Extramedullary leukemia and myeloid sarcoma in AML: new clues to an old issue. Results of a retrospective population-based registry-study of 2261 patients. Accessed April 1, 2021. https://portal.findresearcher.sdu. $\mathrm{dk} /$ en/publications/extramedullary-leukemia-and-myeloid-sarcomain-aml-new-clues-to-a

9. Lee D, Omofoye OA, Nuño MA, Riestenberg RA, Shahlaie K. Treatment outcomes of intracranial myeloid sarcomas: a meta-analysis. World Neurosurg. 2021;148:29-37.

10. Narayanan G, Soman LV, Haridas L, Sugathan H. Isolated intracranial myeloid sarcoma occurring as relapse in acute myeloid leukemia. J Neurosci Rural Pract. 2017;8(3):466-468.

11. Eshghabadi M, Shojania AM, Carr I. Isolated granulocytic sarcoma: report of a case and review of the literature. J Clin Oncol. 1986; 4(6):912-917.

12. Antic D, Verstovsek S, Elezovic I, et al. Spinal epidural granulocytic sarcoma in non-leukemic patient. Int J Hematol. 2009;89(1):95-97.

13. Grier DD, Al-Quran SZ, Gray B, Li Y, Braylan R. Intracranial myeloid sarcoma. Br J Haematol. 2008;142(5):681.

14. Azzarelli V, Roessmann U. Pathogenesis of central nervous system infiltration in acute leukemia. Arch Pathol Lab Med. 1977;101(4): 203-205.

15. lizuka Y, Aiso M, Oshimi K, et al. Myeloblastoma formation in acute myeloid leukemia. Leuk Res. 1992;16(6-7):665-671.

16. Pui C-H, Howard SC. Current management and challenges of malignant disease in the CNS in paediatric leukaemia. Lancet Oncol. 2008;9(3):257-268.

17. Arber DA, Borowitz MJ, Cessna M, et al. Initial diagnostic workup of acute leukemia: guideline from the College of American Pathologists and the American Society of Hematology. Arch Pathol Lab Med. 2017;141(10):1342-1393.

18. Magdy M, Abdel Karim N, Eldessouki I, Gaber O, Rahouma M, Ghareeb M. Myeloid sarcoma. Oncol Res Treat. 2019;42(4): 224-229.

\section{Disclosures}

The authors report no conflict of interest concerning the materials or methods used in this study or the findings specified in this paper.

\section{Author Contributions}

Conception and design: all authors. Acquisition of data: Kohli. Analysis and interpretation of data: Kohli, Koltz. Drafting the article: all authors. Critically revising the article: all authors. Reviewed submitted version of manuscript: Kohli, Koltz. Approved the final version of the manuscript on behalf of all authors: Kamath. Administrative/technical/material support: Kohli, Koltz.

\section{Correspondence}

Ashwin Kamath: Dell Medical School, The University of Texas at Austin, Austin, TX. ashwin.kamath@ascension.org. 